\title{
(G) $\mathfrak{x} \mathfrak{u} \mathfrak{n} \mathfrak{d} \mathfrak{\mathfrak { a }} \mathfrak{\mathfrak { B }} \mathfrak{e}$ \\ .... \\ theoretif(ten und experimentellen \\ $\mathfrak{R} \mathfrak{a} \mathfrak{m} \mathfrak{e} \mathfrak{a} \mathfrak{l}=\mathfrak{C} \mathfrak{l} \mathfrak{e} \mathfrak{m} \mathfrak{i} \mathfrak{e}$
}

zum Bebraudje afabemijajer Borlejungen, fo wie zur Selbftbelebrung,

$\boldsymbol{f} \mathfrak{a} \mathfrak{x}$

Rameraliften, $\mathfrak{A}$ gronomen, Forfibeamte, $\mathfrak{P} 0=$ licevbeante und Tednologen.

$\mathfrak{2} \circ \mathbf{n}$

\section{Sirgegnumo Friedrid) Shermbitádt,}

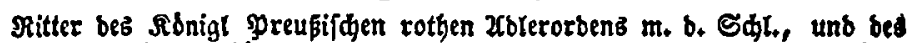

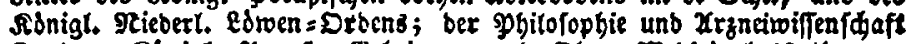
Doctor; Sonigl. Yyreus. Begcimer $=$ und Dber $=$ MRebiginal $=$ SRath; or bentlider dffentlider Eegret ber Ehemie, פharmacie unb Zednotogie

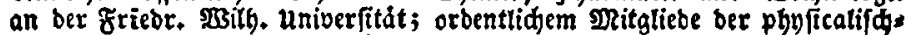

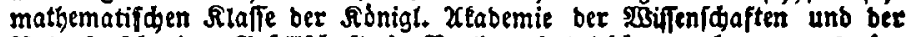

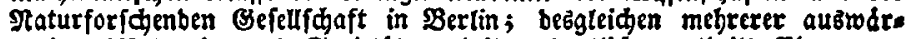

tiger Xeabemien unb Societdten, theris orbentlidem, theild Eghens, theils Eorrefponbirendem IRitgliebe.

Dritte burbaus umgearbeitete und verbefferte Xuflage.

$$
\text { Erfter } \mathfrak{z} \mathfrak{b} \in \mathfrak{i} \mathfrak{l},
$$

welá)e bie vorbercitenben elementaren Senntnifte aus ber

Shģif unb Sbemie entbalt.

\section{$\mathfrak{B} \in \mathfrak{r} \mathfrak{i}$,}

(jetrudt uno verlegt bei (3. Neimer. 



\section{Seiner Maieftát b m}

Nónig der Niederlande

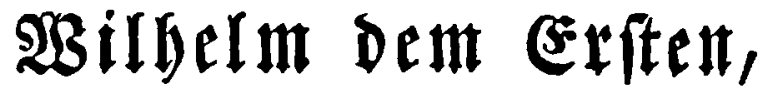
b $\mathrm{m}$

glorreiden Befórderer der 2 Biffenidaften, Sunfte und Gewerbe,

in tiefier Egrfurdt ougeeignet

p 0 a

Dem $\mathfrak{B}$ erfaffer. 
